\title{
K-regularity of locally convex algebras
}

\author{
Hvedri Inassaridze $e^{1,2}$
}

Received: 15 June 2016 / Accepted: 1 July 2016 / Published online: 19 October 2016

(C) Tbilisi Centre for Mathematical Sciences 2016

\begin{abstract}
The isomorphism of Karoubi-Villamayor $K$-groups with smooth $K$-groups for monoid algebras over quasi-stable locally convex algebras is established. We prove that the Quillen $K$-groups are isomorphic to smooth $K$-groups for monoid algebras over quasi-stable Fréchet algebras having a properly uniformly bounded approximate unit and not necessarily $m$-convex. Based on these results the $K$-regularity property for quasi-stable Fréchet algebras having a properly uniformly bounded approximate unit is established.
\end{abstract}

Keywords Smooth K-groups · K-regularity · Properly uniformly bounded approximate unit · Quasi stable locally convex algebra

Mathematics Subject Classification 19K99 - 19D25 · 19D55 · 19L99

Communicated by Tim Porter and George Janelidze.

The author would like to express his thanks to Larry Schweitzer, Guillermo Cortiñas, Jonathan Rosenberg and Ralf Meyer for valuable and useful comments having substantially improved the presentation of this work. This research was supported by Shota Rustaveli National Science Foundation, Grant FR/189/5-113/14.

$凶$ Hvedri Inassaridze inassari@gmail.com

1 A. Razmadze Mathematical Institute of Tbilisi State University, 6, Tamarashvili Str., 0177 Tbilisi, Georgia

2 Tbilisi Centre for Mathematical Sciences, Chavchavadze Ave. 75, 3/35, 0168 Tbilisi, Georgia 


\section{Introduction}

$K$-regularity is an important $K$-theoretical property of rings. It is closely related to the homotopy property of functors and to the Fundamental Theorem of algebraic $K$-theory.

The investigation of $K$-regularity appears in works of Grothendieck for regular rings and is also treated by Bass in [2].

The starting point is the classical Grothendieck-Serre theorem stating that a regular ring is $K_{0}$-regular. This result was further extended by Bass-Heller-Swan for the Whitehead-Bass algebraic $K$-functor $K_{1}$ and by Quillen for all algebraic $K$-functors $K_{n}, n \geq 1$, in [19]. The $K$-regularity property of rings was also introduced and studied by Gersten in [6] and Karoubi in [16].

In this direction more recent results were obtained by Gubeladze for the general case when the rings of polynomials are replaced by monoid rings satisfying certain conditions and the relationship was established between the $K$-regularity of a monoid ring $R$ and its algebraic properties in [7,8]. Cortiñas, Haesemeyer and Weibel have investigated the relationship between K-regularity and regularity for affine schemes confirming a conjecture of Vorst in [5]. For commutative $C^{\star}$-algebras $K$-regularity was established by Rosenberg in [21]. Further results about $K$-regularity of operator algebras were obtained for stable $C^{\star}$-algebras in [10] and for stable profinite $C^{\star}$ algebras in [13].

Our aim is to find a wide class of locally convex algebras for which the $K$-regularity property holds. For this purpose first we will investigate the relationship between Karoubi-Villamayor algebraic $K$-theory and topological $K$-theory in the category of locally convex complex algebras. Then the existing comparison of Quillen algebraic $K$-theory and topological $K$-theory [4,14,22,24,28,29] will be extended to monoid algebras over locally convex algebras. To establish these results the topological invariants introduced in $[14,15]$ and called smooth $K$-groups will be used. It should be noted that $K$-theories related to smooth $K$-groups have been treated in $[4,26]$.

The novelty of our approach allowed us to confirm the Karoubi conjecture for arbitrary Fréchet algebras (not necessarily multiplicatively convex) by using methods of Higson and Suslin-Wodzicki that were not investigated previously for this general case of Fréchet algebras with properly uniformly bounded approximate unit.

A short paper on these results (without proofs) has been published in [11].

\section{Preliminaries}

In this section we recall some definitions and propositions given in [14,15], which will be used later.

By a locally convex algebra we mean an algebra over the field $\mathbb{C}$ of complex numbers equipped with a Hausdorff complete locally convex topology and jointly continuous multiplication The category ALC of locally convex algebras is closed under the Grothendieck projective tensor product [27]. 
Definition 2.1 Let

$$
0 \rightarrow C \stackrel{f}{\rightarrow} B \stackrel{g}{\rightarrow} A \rightarrow 0
$$

be a sequence of morphisms in the category $\mathbf{L C}$ of locally convex linear topological spaces and continuous linear maps. It will be said that this sequence is an exact sequence if $f$ is a homeomorphism of $C$ on $\operatorname{Im} f, g$ is an open surjective map and $\operatorname{Im} f=\operatorname{Ker} g$. It will be said to be proper split exact if it is exact and $g$ has a right inverse in LC. A short exact sequence in the category $\mathbf{A L C}$ is said to be proper exact sequence if it is split exact sequence in the category $\mathbf{L C}$.

Proposition 2.2 If

$$
0 \rightarrow C \rightarrow B \rightarrow A \rightarrow 0
$$

is a proper exact sequence of locally convex algebras and D is a locally convex algebra, then the sequence

$$
0 \rightarrow D \widehat{\otimes} C \rightarrow D \widehat{\otimes} B \rightarrow D \widehat{\otimes} A \rightarrow 0
$$

is a proper exact sequence.

As noted above the smooth $K$-theory was introduced in $[14,15]$ for locally convex algebras. The same definition is valid for arbitrary real or complex topological algebras and is completely similar. Namely, let $A^{\infty(I)}$ be the topological algebra of smooth maps from the unit interval $I$ to the topological algebra $A$. Any continuous homomorphism of topological algebras $\varphi: A \rightarrow A^{\prime}$ induces a homomorphism of topological algebras $\varphi^{\infty(I)}: A^{\infty(I)} \rightarrow A^{\prime \infty(I)}$. For any topological algebra $A$ consider the evaluation maps at $t=0$ and $t=1$

$$
A^{\infty(I)} \rightarrow A, \quad \varepsilon_{0}(f)=f(0), \quad \varepsilon_{1}=f(1)
$$

Denote by $\mathfrak{I}(A)$ the kernel of $\varepsilon_{0}$ and by $\tau_{A}: \mathfrak{I}(A) \rightarrow A$ the restriction of $\varepsilon_{1}$ on $\mathfrak{I}(A)$. There is a smooth homomorphism $\delta_{A}: \mathfrak{I}(A) \rightarrow \mathfrak{I}^{2}(A)$ sending $f \in \mathfrak{I}(A)$ to $\delta_{A}(f)(s, t)=f(s t)$. One gets the smooth path cotriple $\mathfrak{I}$ (for locally convex algebras see [14]) which induces the augmented simplicial group

$$
G L\left(\mathfrak{I}_{*}^{+}(A)\right)=G L\left(\mathfrak{I}_{*}(A)\right) \rightarrow G L(A) .
$$

Definition 2.3 For any topological algebra $A$ the smooth $K$-functors $K_{n}^{\mathrm{sm}}, n \geq 0$, are defined as follows

$$
K_{n}^{\mathrm{sm}}(A)=\pi_{n-2} G L\left(\mathfrak{I}_{*}(A)\right)
$$

for $n \geq 3, K_{0}^{\mathrm{sm}}(A)=K_{0}(A)$ and for $n=1,2$ are defined by the exact sequence

$$
0 \rightarrow K_{2}^{\mathrm{sm}}(A) \rightarrow \pi_{0}\left(G L\left(\mathfrak{I}_{*}(A)\right) \rightarrow G L(A) \rightarrow K_{1}^{\mathrm{sm}}(A) \rightarrow 0 .\right.
$$


All defined smooth $K$-groups are abelian (the proof for the case $n=1,2$ is similar to the case of locally convex algebras [14]).

Let $\mathfrak{F}$ and $\mathfrak{P}$ be the free cotriple and the polynomial cotriple respectively on the category of associative rings and $\mathfrak{J}$ the continuous path cotriple on the category of topological algebras. Then one has natural morphisms

$$
\mathfrak{F} \stackrel{\alpha}{\rightarrow} \mathfrak{P} \stackrel{\delta}{\rightarrow} \mathfrak{I} \stackrel{\beta}{\rightarrow} \mathfrak{J}
$$

For any topological algebra $A$ the homomorphism $\alpha_{A}: \mathfrak{F}(A) \rightarrow \mathfrak{P}(A)$ is given by $\alpha_{A}(|a|)=a x,|a| \in F_{A}$, where $F_{A}$ is the free algebra generated by $A, \delta_{A}(a x)(t \mapsto$ $a t), t \in I$, and one has the inclusion $\beta_{A}: \mathfrak{I}(A) \rightarrow \mathfrak{J}(A)$.

The topological $K$-groups $\left.K_{n}^{\text {top }}(A)=\pi_{n-2} G L\left(\mathfrak{J}_{*}^{+}(A)\right), K_{0}^{\text {top }}(A)=K_{0}(A)\right)$, were defined by Swan in [25] and the algebraic K-groups $K V_{n}(A)=\pi_{n-2} G L\left(\mathfrak{P}_{*}^{+}(A)\right)$ by Karoubi and Villamayor in [17] for $n \geq 1$. The morphisms $\alpha, \delta$ and $\beta$ induce respectively functorial homomorphisms

$$
K_{n}(A) \rightarrow K V_{n}(A) \rightarrow K_{n}^{\mathrm{sm}}(A) \rightarrow K_{n}^{\mathrm{top}}(A)
$$

for $n \geq 1$, where $K_{*}(A)$ are Quillen's $K$-groups, which are isomorphic to Swan's algebraic $K$-functors defined in [25] similarly to the topological case, but with the continuous path cotriple replaced by the free cotriple. These homomorphisms are surjective for $n=1$ (see [12]).

Definition 2.4 Two homomorphisms $f, g: A \rightarrow B$ of topological algebras are said to be smoothly homotopic if there exists a continuous homomorphism $h: A \rightarrow B^{\infty(I)}$ such that $f=\varepsilon_{0} h, g=\varepsilon_{1} h$, which is called a smooth homotopy between $f$ and $g$.

Denote by $\mathbf{G r}$ and $\mathbf{A b}$ the category of groups and abelian groups, respectively.

Definition 2.5 A functor $T: \mathbf{A} \rightarrow \mathbf{G r}$ is called a smooth homotopy functor if $T(f)=$ $T(g)$ for smoothly homotopic $f$ and $g$.

Proposition 2.6 Let $\mathbf{B}$ be a full subcategory of the category $\mathbf{A}$ of topological algebras containing with any topological algebra A the topological algebra $A^{\infty(I)}$. A functor $T: \mathbf{B} \rightarrow \mathbf{G r}$ is a smooth homotopy functor if and only if the inclusion $i: A \rightarrow A^{\infty(I)}$ induces an isomorphism $T(i): T(A) \rightarrow T\left(A^{\infty(I)}\right)$ for any topological algebra $A$ of the category $\mathbf{B}$.

Proposition 2.7 The topological $K$-functors $K_{n}^{\text {top }}$ and the smooth $K$-functors $K_{n}^{\mathrm{sm}}$ are smooth homotopy functors for $n \geq 1$ on the category of topological algebras.

Definition 2.8 Let $(a . u)$ be an approximate unit of a Fréchet algebra $A$. If there exist a Banach algebra $B$ and a continuous injective algebra homomorphism $f: B \rightarrow A$ such that $(a . u)$ is the image of a bounded approximate unit of $B$, then it is called a properly uniformly bounded approximate unit of the Fréchet algebra $A$.

If $A$ is an m-convex Fréchet algebra, then the set $A_{b}$ of uniformly bounded elements in $A$ becomes a Banach algebra with respect to the norm $\|a\|=\sup \|a\|_{n}$. Suppose $A$ 
has a bounded approximate unit in $A_{b}$. Then by considering the inclusion $A_{b} \hookrightarrow A$ and by Definition 2.8 this approximate unit is a properly uniformly bounded approximate unit and in this particular case it is called uniformly bounded approximate unit of $A$.

If $A$ is an arbitrary unital Fréchet algebra with the sequence of determining seminorms $\|\cdot\|_{n}$, then with respect to the inclusion $\mathbb{C} e \hookrightarrow A$ the unit $e$ is a properly uniformly bounded approximate unit of $A$. Note that the unit $e$ may be unbounded in $A$, depending on the given sequence of determining seminorms.

The Fréchet algebras considered in this article are not necessarily locally multiplicatively convex (m-convex). Finite projective tensor products of Fréchet algebras with properly uniformly bounded approximate unit are again Fréchet algebras with properly uniformly bounded approximate unit. The unit element of any unital Fréchet algebra $A$ is a properly uniformly bounded approximate unit of $A$. Projective limits of countably many $C^{*}$-algebras are Fréchet algebras with properly uniformly bounded approximate unit.

Many important examples of functional algebras in analysis are not locally multiplicatively convex and are Fréchet algebras with properly uniformly bounded approximate unit (for instance the Arens algebra of complex-valued measurable functions on the unit interval [1], Schwartz convolution group algebras [23], Theorem 3.2.8 and Proposition 3.2.13; the algebra of functions holomorphic on the unit disk [30], Theorem 26). Examples of non - m-convex Fréchet algebras are also given in $[18,20]$. If $A$ is a Fréchet algebra with properly uniformly bounded approximate unit, then so is the Fréchet algebra $A^{\infty(I)}$.

The main and needed $K$-theoretical property of Fréchet algebras with properly uniformly bounded approximate unit is expressed in the following assertion:

Theorem 2.9 If A is a Fréchet algebra with properly uniformly bounded approximate unit and not necessarily $m$-convex, then it possesses the TF-property and therefore the excision property in algebraic $K$-theory and the $H$-unitality property [14].

\section{Smooth Karoubi conjecture and K-regularity}

First we recall the notion of $K$-regularity property for associative rings.

Definition 3.1 A ring $A$ is called $K$-regular if the natural injection $A \rightarrow A\left[x_{1}, x_{2}\right.$, $\left.\ldots, x_{m}\right]$ induces an isomorphism

$$
K_{n}(A) \cong K_{n}\left(A\left[x_{1}, x_{2}, \ldots, x_{m}\right]\right)
$$

for all $n, m>0$.

Besides the aforementioned assertions given in [14] for locally convex algebras we will use the following important property of smooth maps:

Lemma 3.2 There is an isomorphism

$$
A^{\infty(I)} \widehat{\otimes} B \cong(A \widehat{\otimes} B)^{\infty(I)}
$$

for any locally convex algebras $A$ and $B$. 
Proof A minor generalization of Theorem 44.1 [27] shows that $\mathbb{C}^{\infty(I)} \widehat{\otimes}_{\epsilon} A \cong A^{\infty(I)}$, where $\widehat{\otimes}_{\epsilon}$ is the completed $\epsilon$-tensor product. By the nuclearity of $\mathbb{C}^{\infty(I)}$, Theorem 50.1 [27] says that $\mathbb{C}^{\infty(I)} \widehat{\otimes} A \cong \mathbb{C}^{\infty(I)} \widehat{\otimes}_{\epsilon} A$. Finally, one gets

$$
A^{\infty(I)} \widehat{\otimes} B \cong\left(\mathbb{C}^{\infty(I)} \widehat{\otimes} A\right) \widehat{\otimes} B \cong \mathbb{C}^{\infty(I)} \widehat{\otimes}(A \widehat{\otimes} B) \cong(A \widehat{\otimes} B)^{\infty(I)} .
$$

This completes the proof.

Remark 3.3 (1) For $m$-convex locally convex algebras Lemma 3.2 is proved in [27]. The proof for the general case is due to Larry Schweitzer.

(2) It should be noted that this property does not hold for continuous maps and arbitrary locally convex algebras, even for Banach algebras. That is the reason for which we have introduced smooth $K$-groups and smooth homotopy functors instead of topological $K$-groups and homotopy functors respectively for the investigation of Karoubi conjecture about the isomorphism of algebraic and topological $K$-functors in the case of locally convex algebras, which we call the smooth Karoubi conjecture (see [14,15]).

(3) This isomorphism also holds when $(-)^{\infty(I)}$ is replaced by $\mathfrak{I}(-)$.

Theorem 3.4 Let B be a full subcategory of the category A of topological algebras containing with any topological algebra $A$ the topological algebra $A^{\infty(I)}$. Then

(1) the functors $K_{1}$ and $K_{1}^{\mathrm{sm}}$ are isomorphic on the category $\mathbf{B}$ if and only if $K_{1}$ is a smooth homotopy functor on $\mathbf{B}$,

(2) the functors $K V_{1}$ and $K_{1}^{\mathrm{sm}}$ are isomorphic on the category $\mathbf{B}$ if and only if $K V_{1}$ is a smooth homotopy functor on $\mathbf{B}$.

Proof (1) Let $K_{1}$ and $K_{1}^{\mathrm{sm}}$ be isomorphic functors on B. Consider for any topological algebra $A$ the following commutative diagram

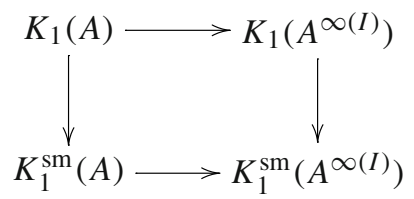

where the vertical homomorphisms and the bottom homomorphism are isomorphisms. Hence so is the top homomorphism. Now Proposition 2.6 shows the smooth homotopy property of $K_{1}$.

Let $K_{1}$ be a smooth homotopy functor on $\mathbf{B}$. The surjection $\mathfrak{I}(A) \rightarrow A$ induces the following commutative diagram with exact rows and columns 


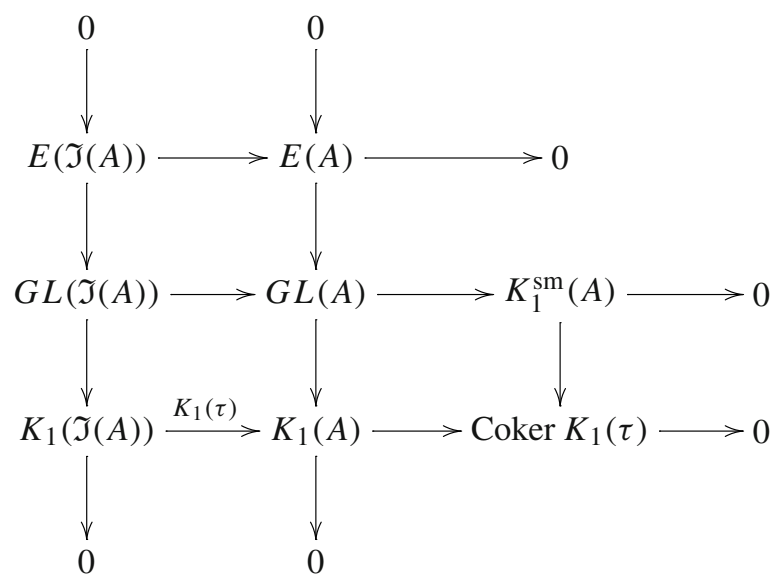

which implies the isomorphism $K_{1}^{s m}(A) \cong \operatorname{Coker} K_{1}(\tau)$.

Now we will use the notion of smooth homotopization $h^{\mathrm{sm}} T$ of any functor $T$ : $\mathbf{A} \rightarrow \mathbf{G r}$ defined by

$$
h^{\mathrm{sm}} T(A)=\operatorname{Coker}\left(T\left(A^{\infty(I)}\right) \rightrightarrows T(A)\right)
$$

where Coker denotes the coequaliser of these two homomorphisms induced by evaluation maps. By using the exactness property of the functor $K_{1}$, it is easily shown that

$$
\left(h^{\mathrm{sm}} K_{1}\right)(A) \approx \operatorname{Coker}(\tau) .
$$

Thus one gets the isomorphism $K_{1}^{\mathrm{sm}}(A) \approx h^{\mathrm{sm}} K_{1}(A)$. It follows that if $K_{1}(A) \approx$ $K_{1}\left(A^{\infty(I)}\right)$ one obtains the required isomorphism.

(2) The first part of the proof is similar to the case 1. Let $K V_{1}$ be a smooth homotopy functor on B. Since $K_{1}^{\mathrm{sm}}$ is a smooth homotopy functor, the homomorphism $\delta_{1}$ : $K V_{1}(A) \rightarrow K_{1}^{s m}(A)$ just induces a homomorphism $h^{s m} \delta_{1}:\left(h^{s m}\left(K V_{1}\right)\right)(A) \rightarrow$ $K_{1}^{s m}(A)$.

The commutative diagram

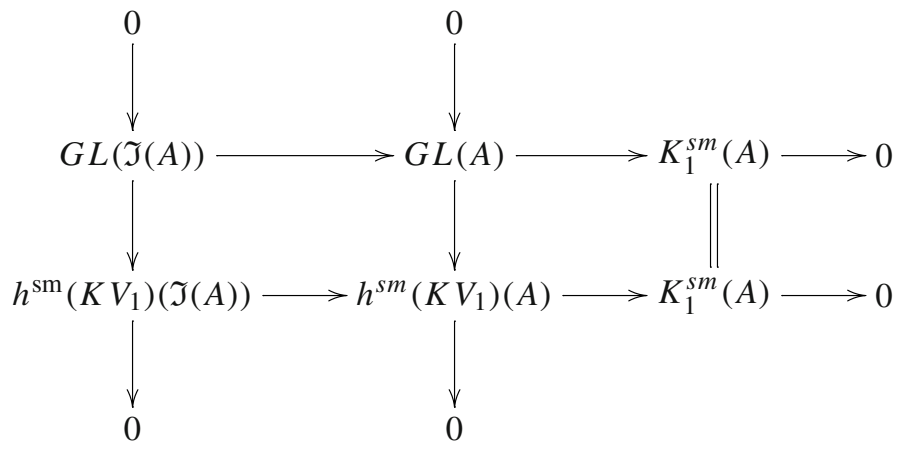


with exact top row and vertical surjective homomorphisms implies the exactness of the bottom row. The topological algebra $\mathfrak{I}(A)$ is contractible because the trivial map $0_{\mathfrak{I}(A)}$ and the identity map $1_{\mathfrak{I}(A)}$ are smoothly homotopic with smooth homotopy $\delta_{A}: \mathfrak{I}(A) \rightarrow \mathfrak{I}^{2}(A)$ between them. Since $h^{\mathrm{sm}}\left(K V_{1}\right)$ is a smooth homotopy functor, one has $h^{\mathrm{sm}}\left(K V_{1}\right)(\mathfrak{I}(A))=0$ implying the isomorphism $h^{\mathrm{sm}}\left(K V_{1}\right)(A) \rightarrow K_{1}^{\mathrm{sm}}(A)$. Thus $K V_{1}(A) \rightarrow K_{1}^{\mathrm{sm}}(A)$ is an isomorphism if $K V_{1}(A) \rightarrow K V_{1}\left(A^{\infty(I)}\right)$ is an isomorphism. This completes the proof.

Later this result will be applied just to monoid algebras over quasi-stable locally convex algebras.

Let $M$ be a monoid and $A[M]$ a monoid algebra over a locally convex algebra $A$. Each determining seminorm $\mu$ of $A$ induces in a natural way a seminorm $\mu_{M}$ on $A[M]$ as follows

$$
\mu_{M}\left(\sum a_{j} m_{j}\right)=\sum \mu\left(a_{j}\right)
$$

for any element $\sum a_{j} m_{j}$ of $A[M]$. Then $A[M]$ becomes a topological algebra with respect to these induced seminorms. Regarding the topology on the monoid algebra $A[M]$ over a locally convex algebra $A$, it can be considered as the union of the set $S$ of finite products of copies of $A$ indexed by finite subsets of $M$ and partially ordered by inclusion. Then we take on $A[M]$ the union topology induced by the topology of these finite products.

To confirm Karoubi's conjecture about the isomorphism of algebraic and topological $K$-functors we need the following important triple factorization property introduced in [24]. We recall its definition.

Definition 3.5 It is said that a ring $A$ possesses the property $(T F)_{\text {right }}$ if for any finite collection of elements $a_{1}, a_{2}, \ldots, a_{m}$ of the ring $A$ there exist elements $b_{1}, b_{2}, \ldots, b_{m}, c, d \in A$ such that $a_{i}=b_{i} c d$ for $1 \leq i \leq m$ and the left annihilators in $A$ of $c$ and $c d$ are equal.

Proposition 3.6 If a ring A has the $(T F)_{\text {right }}$ property, then the monoid algebra $A[M]$ has the $(T F)_{\text {right }}$ property for any monoid $M$.

Proof Let

$$
\begin{gathered}
p_{1}=a_{11} m_{01}+a_{12} m_{11}+\cdots+a_{1 n_{1}} m_{1 n_{1}} \\
p_{2}=a_{21} m_{21}+a_{22} m_{22}+\cdots+a_{2 n_{2}} m_{2 n_{2}} \\
\ldots \ldots \ldots \ldots \ldots \ldots \ldots \ldots \ldots \ldots \ldots \ldots \\
p_{k}=a_{k 1} m_{k 2}+a_{k 2} m_{k 2}+\cdots+a_{k n_{k}} m_{k n_{k}}
\end{gathered}
$$

be elements of the monoid algebra $A[M]$. The $(T F)_{\text {right }}$ property of the ring $A$ implies that for the elements

$$
a_{11}, \ldots, a_{1 n_{1}}, a_{21}, \ldots, a_{2 n_{2}}, \ldots, a_{k 1}, \ldots, a_{k n_{k}}
$$


of the ring $A$ there exist elements

$$
b_{11}, \ldots, b_{1 n_{1}}, b_{22}, \ldots, b_{2 n_{2}}, \ldots, b_{k 1}, \ldots, b_{k n_{k}}
$$

and $c, d$ of $A$ such that

$$
\begin{gathered}
a_{1 j}=b_{1 j} c d, j=1, \ldots, n_{1} \\
a_{2 j}=b_{2 j} c d, j=1, \ldots, n_{2} \\
\ldots \ldots \ldots \ldots \ldots, \ldots \ldots, \ldots \\
a_{k j}=b_{k j} c d, j=1, \ldots, n_{k}
\end{gathered}
$$

and if $y c d=0$ then $y c=0$ for $y \in A$.

Now consider the following elements of the monoid algebra $A[M]$ :

$$
\begin{gathered}
q_{1}=b_{11} m_{01}+b_{12} m_{11}+\cdots+b_{1 n_{1}} m_{1 n_{1}} \\
q_{2}=b_{21} m_{21}+b_{22} m_{22}+\cdots+b_{2 n_{2}} m_{2 n_{2}} \\
\ldots \ldots \ldots \ldots \ldots \ldots \ldots \ldots+\cdots \cdots \cdots \\
q_{k}=b_{k 1} m_{k 2}+b_{k 2} m_{k 2}+\cdots+b_{k n_{k}} m_{k n_{k}}
\end{gathered}
$$

It is clear that one has $p_{i}=q_{i} c d$ for $1 \leq i \leq k$. If $p c d=0$ for some monoid algebra element $p=a_{1} m_{1}+a_{2} m_{2}+\cdots+a_{n} m_{n}$, then $a_{j} c d=0$ for $0 \leq j \leq n$. Thus one gets $a_{j} c=0,0 \leq j \leq n$, implying $p c=0$. Therefore the monoid algebra $A[M]$ has the $(T F)_{\text {right }}$ property.

This proposition generalizes Lemma 16 [10]. Therefore the polynomial algebra $A\left[x_{1}, x_{2}, \ldots, x_{m}\right], n \geq 1$, and the Laurent polynomial algebra $A\left[t, t^{-1}\right]$ over a Fréchet algebra $A$ with properly uniformly bounded approximate unit possess the excision property in algebraic $K$-theory and the $H$-unitality property.

In what follows the following property of smooth maps will be used

$$
(A[M])^{\infty(I)} \approx\left(A^{\infty(I)}\right)[M]
$$

for any locally convex algebra algebra $A$, which is in particular satisfied if the monoid $M$ is countable. It is clear that this condition implies the isomorphism $\mathfrak{I}(A[M]) \approx$ $(\mathfrak{I}(A))[M]$ too.

Let $M$ be a monoid and denote by ALC $[M]$ the category of monoid algebras $A[M]$ over locally convex algebras $A$ and by $\mathbb{C}^{\star}$ the category of $C^{\star}$-algebras. Let $T$ be an arbitrary functor $\mathbf{T}$ from the category $\mathbf{A L C}[M]$ to the category $\mathbf{A b}$ of abelian groups.

Definition 3.7 A locally convex algebra $B$ is called quasi-stable if it has the form $A \widehat{\otimes} \mathcal{K}$ for some locally convex algebra $A$ where $\mathcal{K}$ is the $C^{\star}$-algebra of compact operators on the infinite dimensional Hilbert space $\mathcal{H}$.

Theorem 3.8 If the functor

$$
T((A \widehat{\otimes}(-\otimes \mathcal{K}))[M]): \mathbb{C}^{\star} \rightarrow \mathbf{A b}
$$


is a stable and split exact functor for any fixed locally convex algebra A of a full subcategory $\mathbf{B}$ of $\mathbf{A L C}$, then the functor

$$
T((-\widehat{\otimes} \mathcal{K})[M]): \mathbf{B} \rightarrow \mathbf{A b}
$$

is a smooth homotopy functor.

Proof The Higson homotopy invariance theorem [9], which is true for complex (or real) $C^{\star}$-algebras, implies that the functor

$$
T((A \widehat{\otimes}(-\otimes \mathcal{K}))[M])): \mathbb{C}^{\star} \rightarrow \mathbf{A b}
$$

is homotopy invariant.

By using Lemma 3.2 one can create the following commutative diagram for any $C^{\star}$-algebra $D$

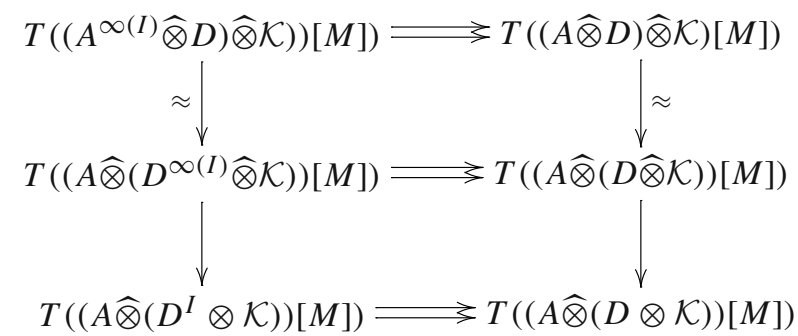

where the horizontal maps are induced by evaluation maps. The homotopy invariance of the functor $T((A \widehat{\otimes}(-\otimes \mathcal{K}))[M])$ implies the equality of the bottom two horizontal homomorphisms and therefore the equality of the top two horizontal homomorphisms. Therefore, this equality holds for any locally convex algebra of the subcategory $\mathbf{B}$ and we obtain the desired smooth homotopy invariance of the functor $T((-\widehat{\otimes} \mathcal{K})[M])$. This completes the proof.

Theorem 3.9 For any locally convex algebra A there is an isomorphism

$$
K V_{n}((A \widehat{\otimes} \mathcal{K})[M]) \rightarrow K_{n}^{\mathrm{sm}}((A \widehat{\otimes} \mathcal{K})[M])
$$

for all $n \geq 1$.

Proof First it will be shown that $K V_{n}((-\widehat{\otimes} \mathcal{K})[M]), n \geq 1$, is a smooth homotopy functor on the category of locally convex algebras. According to Theorem 3.8 it suffices to prove that the functor $K V_{n}(((A \widehat{\otimes}(-\otimes \mathcal{K}))[M]), n \geq 1$, is stable and split exact on the category of $\mathrm{C}^{*}$-algebras for any locally convex algebra $A$.

Let

$$
0 \rightarrow D_{1} \rightarrow D \rightarrow D_{2} \rightarrow 0
$$

be a split exact sequence of $\mathrm{C}^{*}$-algebras. Then the sequence

$$
0 \rightarrow\left(A \widehat{\otimes}\left(D_{1} \otimes \mathcal{K}\right)\right)[M] \rightarrow(A \widehat{\otimes}(D \otimes \mathcal{K}))[M] \rightarrow\left(A \widehat{\otimes}\left(D_{2} \otimes \mathcal{K}\right)\right)[M] \rightarrow 0
$$


is also a split exact sequence for any locally convex algebra $A$. It is well known that any short split exact sequence of rings is a $G L$-fibration with respect to polynomial cotriple. Therefore Karoubi-Villamayor algebraic K-functors preserve short split exact exactness of rings and it follows that the sequence

$$
\begin{aligned}
0 & \rightarrow K V_{n}\left(\left(A \widehat{\otimes}\left(D_{1} \otimes \mathcal{K}\right)\right)[M]\right) \rightarrow K V_{n}((A \widehat{\otimes}(D \otimes \mathcal{K}))[M]) \\
& \rightarrow K V_{n}\left(\left(A \widehat{\otimes}\left(D_{2} \otimes \mathcal{K}\right)\right)[M]\right) \rightarrow 0
\end{aligned}
$$

is a short split exact sequence of abelian groups.

Let $D$ be a $C^{\star}$-algebra. Then for any locally convex algebra $A$ the canonical homomorphism $D \rightarrow D \otimes \mathcal{K}$ induces the homomorphism

$$
K V_{n}((A \widehat{\otimes}(D \otimes \mathcal{K}))[M]) \rightarrow K V_{n}((A \widehat{\otimes}((D \otimes \mathcal{K}) \otimes \mathcal{K})[M])
$$

On the other hand one has natural isomorphisms

$$
\begin{aligned}
& K V_{n}\left(( A \widehat { \otimes } ( ( D \otimes \mathcal { K } ) \otimes \mathcal { K } ) [ M ] ) \cong K V _ { n } \left(\left(A \widehat{\otimes}\left(D \otimes M_{2}(\mathcal{K})\right)[M]\right)\right.\right. \\
& \quad \cong K V_{n}\left(\left(A \widehat{\otimes} M_{2}(D \otimes \mathcal{K})\right)[M]\right) \cong K V_{n}\left(\left(M_{2}(A \widehat{\otimes}(D \otimes \mathcal{K}))[M]\right)\right. \\
& \quad \cong K V_{n}\left(\left(M_{2}(A \widehat{\otimes}(D \otimes \mathcal{K}))[M]\right) .\right.
\end{aligned}
$$

and the composite of the induced homomorphism (3.4) with theses isomorphisms gives us the natural homomorphism

$$
K V_{n}((A \widehat{\otimes}(D \otimes \mathcal{K}))[M]) \rightarrow K V_{n}\left(\left(M_{2}(A \widehat{\otimes}(D \otimes \mathcal{K}))[M]\right)\right.
$$

It is well known [9] that for any ring $B$ one has the isomorphism

$$
K V_{1}(B) \rightarrow K V_{1}\left(M_{2}(B)\right)
$$

implying this isomorphism for all $\mathrm{K}$-functors $K V_{n}, n \geq 1$. Therefore the homomorphism

$$
K V_{n}((A \widehat{\otimes}(D \otimes \mathcal{K}))[M]) \rightarrow K V_{n}\left(M_{2}((A \widehat{\otimes}(D \otimes \mathcal{K}))[M])\right.
$$

is an isomorphism for all $n \geq 1$ and we conclude that the induced homomorphism (3.4) is an isomorphism for all $n \geq 1$. Thus by Theorem 3.8 the functor $K V_{n}((-\widehat{\otimes} \mathcal{K})[M])$, for all $n \geq 1$, is a smooth homotopy functor on the category of locally convex algebras. Taking into account Lemma 3.2 we finally conclude that for any monoid $M$ [satisfying (3.1)] the functor $K V_{n}$ is a smooth homotopy functor on the category of monoid algebras over quasi-stable locally convex algebras for all $n \geq 1$.

The exact sequence

$$
0 \rightarrow \Omega_{\mathrm{sm}}(A) \rightarrow \Im(A) \rightarrow A \rightarrow 0
$$


induces the exact sequence

$$
0 \rightarrow\left(\Omega_{\mathrm{sm}}(A) \widehat{\otimes} \mathcal{K}\right)[M] \rightarrow(\Im(A) \widehat{\otimes} \mathcal{K})[M] \rightarrow(A \widehat{\otimes} \mathcal{K})[M] \rightarrow 0 .
$$

Since $\Im(A) \widehat{\otimes} \mathcal{K})[M]$ is isomorphic to $\Im(A \widehat{\otimes} \mathcal{K})[M])$, this sequence is a $G L$-fibration with respect to the smooth cotriple and therefore with respect to the polynomial cotriple. Thus the sequence (3.4) induces the following long exact sequences

$$
\begin{aligned}
\cdots & \rightarrow K_{n+1}^{\mathrm{sm}}((A \widehat{\otimes} \mathcal{K})[M]) \rightarrow K_{n}^{\mathrm{sm}}\left(\left(\Omega_{\mathrm{sm}}(A) \widehat{\otimes} \mathcal{K}\right)[M]\right) \rightarrow K_{n}^{\mathrm{sm}}((\Im(A) \widehat{\otimes} \mathcal{K})[M]) \\
& \rightarrow K_{n}^{\mathrm{sm}}((A \widehat{\otimes} \mathcal{K})[M]) \rightarrow K_{n-1}^{\mathrm{sm}}\left(\left(\Omega_{\mathrm{sm}}(A) \widehat{\otimes} \mathcal{K}\right)[M]\right) \rightarrow \cdots, \\
\cdots & \rightarrow K V_{n+1}((A \widehat{\otimes} \mathcal{K})[M]) \rightarrow K V_{n}\left(\left(\Omega_{\mathrm{sm}}(A) \widehat{\otimes} \mathcal{K}\right)[M]\right) \rightarrow K V_{n}((\Im(A) \widehat{\otimes} \mathcal{K})[M]) \\
& \rightarrow K V_{n}((A \widehat{\otimes} \mathcal{K})[M]) \rightarrow K V_{n-1}\left(\left(\Omega_{\mathrm{sm}}(A) \widehat{\otimes} \mathcal{K}\right)[M]\right) \rightarrow \cdots .
\end{aligned}
$$

As we have seen the locally convex algebra $\mathfrak{I}(A)$ is smoothly contractible. This imply the contractibility of $(\Im(A) \widehat{\otimes} \mathcal{K})[M]$. Since $K_{n}^{\mathrm{sm}}$ and $K V_{n}$ are smooth homotopy functors on the category of monoid algebras over quasi-stable locally convex algebras, one gets the equalities $\left.K_{n}^{\mathrm{sm}}(\Im(A) \widehat{\otimes} \mathcal{K})[M]\right)=0$ and $\left.K V_{n}(\Im(A) \widehat{\otimes} \mathcal{K})[M]\right)=0$ for all $n \geq 1$.

Therefore the above long exact sequences yield the following isomorphisms

$$
\begin{aligned}
& K_{n}^{\mathrm{sm}}((A \widehat{\otimes} \mathcal{K})[M]) \cong K_{1}^{\mathrm{sm}}\left(\left(\Omega_{\mathrm{sm}}^{n-1}(A) \widehat{\otimes} \mathcal{K}\right)[M]\right), \\
& K V_{n}((A \widehat{\otimes} \mathcal{K})[M]) \cong K V_{1}\left(\left(\Omega_{\mathrm{sm}}^{n-1}(A) \widehat{\otimes} \mathcal{K}\right)[M]\right)
\end{aligned}
$$

for all $n>1$, where $\Omega_{\mathrm{sm}}^{n}(A)=\Omega_{\mathrm{sm}}^{1}\left(\Omega_{\mathrm{sm}}^{n-1}(A)\right)$.

By Theorem 3.4 the abelian groups $K_{1}^{\mathrm{sm}}\left(\left(\Omega_{\mathrm{sm}}^{n-1}(A) \widehat{\otimes} \mathcal{K}\right)[M]\right)$ and $K V_{1}\left(\left(\Omega_{\mathrm{sm}}^{n-1}(A) \widehat{\otimes} \mathcal{K}\right)[M]\right)$ are isomorphic and finally we obtain the required isomorphism:

$$
K^{\mathrm{sm}} n((A \widehat{\otimes} \mathcal{K})[M]) \cong K V_{n}((A \widehat{\otimes} \mathcal{K})[M])
$$

for all $n \geq 1$. This completes the proof.

Theorem 3.9 generalizes Higson's result [9] on the isomorphism of KaroubiVillamayor algebraic K-functors and topological K-functors for stable $C^{\star}$-algebras, since in this case the smooth $\mathrm{K}$-functors are isomorphic to topological K-functors (see Theorem 1.10, [14]).

Theorem 3.10 For any Fréchet algebra A with properly uniformly bounded approximate unit and not necessarily $m$-convex there is an isomorphism

$$
K_{n}((A \widehat{\otimes} \mathcal{K})[M]) \rightarrow K_{n}^{\mathrm{sm}}((A \widehat{\otimes} \mathcal{K})[M])
$$

for all $n \geq 1$. 
Proof To prove that $K_{n}((A \widehat{\otimes}(-\otimes \mathcal{K}))[M]), n \geq 1$, is a smooth homotopy functor on the category of Fréchet algebras with properly uniformly bounded approximate unit we will again use Theorem 3.8 by taking for the functor $T$ the Quillen algebraic $\mathrm{K}$-functor $K_{n}$. Consider the short exact sequences (3.2) and (3.3). The Fréchet algebra $A \widehat{\otimes}\left(C_{1} \otimes \mathcal{K}\right)$ has a properly uniformly bounded approximate unit and consequently by Propostion 3.6 and Theorem 2.9 the monoid algebra $\left(A \widehat{\otimes}\left(C_{1} \otimes \mathcal{K}\right)\right)[M]$ has the excision property in algebraic $\mathrm{K}$-theory and is $\mathrm{H}$-unital. Thus the split exact sequence (3.2) induces the split exact sequence

$$
\begin{aligned}
0 & \rightarrow K_{n}\left(\left(A \widehat{\otimes}\left(C_{1} \otimes \mathcal{K}\right)\right)[M]\right) \rightarrow K_{n}((A \widehat{\otimes}(C \otimes \mathcal{K}))[M]) \\
& \rightarrow K_{n}\left(\left(A \widehat{\otimes}\left(C_{2} \otimes \mathcal{K}\right)\right)[M]\right) \rightarrow 0 .
\end{aligned}
$$

Since $(A \widehat{\otimes}(D \otimes \mathcal{K}))[M]$ is H-unital for any Fréchet algebra $A$ with properly uniformly bounded approximate unit and any $\mathrm{C}^{*}$-algebra $D$, it has the Morita equivalence property implying the isomorphism

$$
K_{n}((A \widehat{\otimes}(D \otimes \mathcal{K}))[M]) \rightarrow K_{n}\left(M_{2}((A \widehat{\otimes}(D \otimes \mathcal{K}))[M])\right.
$$

induced by the canonical homomorphism $D \rightarrow D \otimes \mathcal{K}$. Therefore, by Theorem 3.8 the functor $K_{n}((-\widehat{\otimes} \mathcal{K})[M]), n \geq 1$, is a smooth homotopy functor on the category of Fréchet algebras with properly uniformly bounded approximate unit. Applying now Lemma 3.2 we show that the Quillen algebraic K-functor $K_{n}$ is a smooth homotopy functor on the category of monoid algebras over quasi-stable Fréchet algebras with properly uniformly bounded approximate unit for all $n \geq 1$.

If $A$ is a Fréchet algebra with properly uniformly bounded approximate unit, then $\Omega_{\mathrm{sm}}(A) \widehat{\otimes} \mathcal{K}$ is also a Fréchet algebra with properly uniformly bounded approximate unit. Thus the algebra $\left(\Omega_{\mathrm{sm}}(A) \widehat{\otimes} \mathcal{K}\right)[M]$ has the excision property in algebraic $\mathrm{K}$ theory. It follows that the short exact sequence (3.5) induces the following long exact sequence

$$
\begin{aligned}
\cdots & \rightarrow K_{n+1}((A \widehat{\otimes} \mathcal{K})[M]) \rightarrow K_{n}\left(\left(\Omega_{\mathrm{sm}}(A) \widehat{\otimes} \mathcal{K}\right)[M]\right) \rightarrow K_{n}((\Im(A) \widehat{\otimes} \mathcal{K})[M]) \\
& \rightarrow K_{n}((A \widehat{\otimes} \mathcal{K})[M]) \rightarrow K_{n-1}\left(\left(\Omega_{\mathrm{sm}}((A \widehat{\otimes} \mathcal{K})[M]) \rightarrow \cdots\right.\right.
\end{aligned}
$$

Since $K_{n}$ is a smooth homotopy functor, one has the equality $K_{n}((\Im(A) \widehat{\otimes} \mathcal{K})[M])=0$ for $n \geq 1$ which implies the isomorphism

$$
K_{n}((A \widehat{\otimes} \mathcal{K})[M]) \cong K_{1}\left(\left(\Omega_{\mathrm{sm}}^{n-1}(A \widehat{\otimes} \mathcal{K})[M]\right), \quad n>1\right.
$$

The group $K_{1}\left(\left(\Omega_{s m}^{n-1}(A) \widehat{\otimes} \mathcal{K}\right)[M]\right)$ is isomorphic to the group $K_{1}^{\mathrm{sm}}\left(\left(\Omega_{\mathrm{sm}}^{n-1}(A) \widehat{\otimes} \mathcal{K}\right)[M]\right)$ by Theorem 3.4 and the composition of these two isomorphisms with the isomorphism

$$
K_{1}^{\mathrm{sm}}\left(\left(\Omega_{\mathrm{sm}}^{n-1}(A) \widehat{\otimes} \mathcal{K}\right)[M]\right) \cong K_{n}^{\mathrm{sm}}((A \widehat{\otimes} \mathcal{K})[M])
$$


gives us the required isomorphism

$$
K_{n}^{\mathrm{sm}}((A \widehat{\otimes} \mathcal{K})[M]) \cong K_{n}((A \widehat{\otimes} \mathcal{K})[M])
$$

for all $n \geq 1$. This completes the proof.

Theorem 3.11 For any Fréchet algebra A with properly uniformly bounded approximate unit and not necessarily m-convex one has isomorphisms

$$
K_{n}(A \widehat{\otimes} \mathcal{K}) \cong K_{n}\left((A \widehat{\otimes} \mathcal{K})\left[x_{1}, x_{2}, \ldots, x_{m}\right]\right)
$$

for $n, m \geq 1$.

Proof Consider the following commutative diagram

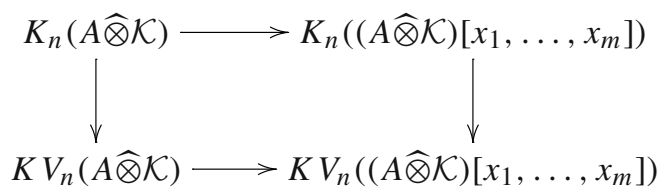

with natural homomorphisms. According to Theorems 3.9 and 3.10 the Quillen algebraic K-groups $K_{n}((A \widehat{\otimes} \mathcal{K})[M])$ are isomorphic to Karoubi-Villamayor algebraic K-groups $K V_{n}((A \widehat{\otimes} \mathcal{K})[M])$ for monoid algebras over quasi-stable Fréchet algebras with properly uniformly bounded approximate unit for $n \geq 1$. Therefore, the vertical homomorphisms are isomorphisms. Since the bottom homomorphism is an isomorphism for any ring, we conclude that the top homomorphism is an isomorphism too. This completes the proof.

Corollary 3.12 Let A be a Fréchet algebra with properly uniformly bounded approximate unit. Then one has isomorphisms

$$
K_{n}\left((A \widehat{\otimes} \mathcal{K})\left[t, t^{-1}\right]\right) \cong K_{n}(A \widehat{\otimes} \mathcal{K}) \oplus K_{n-1}(A \widehat{\otimes} \mathcal{K})
$$

and

$$
K_{n}^{s m}\left((A \widehat{\otimes} \mathcal{K})\left[t, t^{-1}\right]\right) \cong K_{n}^{\mathrm{sm}}(A \widehat{\otimes} \mathcal{K}) \oplus K_{n-1}^{\mathrm{sm}}(A \widehat{\otimes} \mathcal{K})
$$

for all $n \geq 1$.

Proof Denote $B=A \widehat{\otimes} \mathcal{K}$ and let $B^{+}$be the unital Fréchet algebra obtained by adding the unit to $B$. The following split exact sequences

$$
\begin{aligned}
& 0 \rightarrow B \rightarrow B^{+} \rightarrow \mathbb{C} \rightarrow 0, \\
& 0 \rightarrow B[t] \rightarrow B^{+}[t] \rightarrow \mathbb{C}[t] \rightarrow 0
\end{aligned}
$$


and

$$
0 \rightarrow B\left[t, t^{-1}\right] \rightarrow B^{+}\left[t, t^{-1}\right] \rightarrow \mathbb{C}\left[t, t^{-1}\right] \rightarrow 0
$$

induce the split exact sequences of K-groups

$$
\begin{aligned}
& 0 \rightarrow K_{n}(B) \rightarrow K_{n}\left(B^{+}\right) \rightarrow K_{n}(\mathbb{C}) \rightarrow 0, \\
& 0 \rightarrow K_{n}(B[t]) \rightarrow K_{n}\left(B^{+}[t]\right) \rightarrow K_{n}(\mathbb{C}[t]) \rightarrow 0, \\
& 0 \rightarrow K_{n}\left(B\left[t, t^{-1}\right]\right) \rightarrow K_{n}\left(B^{+}\left[t, t^{-1}\right]\right) \rightarrow K_{n}\left(\mathbb{C}\left[t, t^{-1}\right]\right) \rightarrow 0
\end{aligned}
$$

for $n \geq 0$, since the algebras $B, B[t]$ and $B\left[t, t^{-1}\right]$ have the excision property in algebraic K-theory.

If we consider the commutative diagram

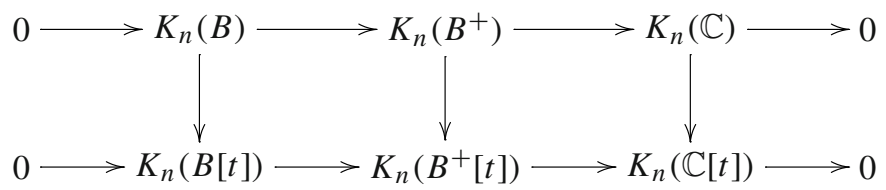

with vertical homomorphisms of K-groups induced by natural injections, we conclude that the middle vertical homomorphism is an isomorphism, since the right vertical one is a well known isomorphism and the other left vertical homomorphism is an isomorphism too by Theorem 3.11

Thus the fundamental theorem of algebraic K-theory gives us the following isomorphisms

$$
\begin{aligned}
& K_{n}\left(B^{+}\left[t, t^{-1}\right]\right) \cong K_{n}\left(B^{+}\right) \oplus K_{n-1}\left(B^{+}\right), \\
& K_{n}\left(\mathbb{C}\left[t, t^{-1}\right]\right) \cong K_{n}(\mathbb{C}) \oplus K_{n-1}(\mathbb{C})
\end{aligned}
$$

for $n \geq 1$. From these isomorphisms and the above obtained short split exact sequences of $K$-groups immediately follows the required first isomorphism of the corollary. The second isomorphism is a consequence of the first isomorphism and Theorem 3.10. This completes the proof.

Remark 3.13 G. Cortiñas let me know that in particular cases, namely for locally multiplicatively convex algebras and countable monoid $M$, Theorems 3.8 and 3.9 can be obtained respectively by Theorem 6.2.1 in [4] and for m-convex Fréchet algebra with uniformly bounded approximate unit by applying argument of Theorem 12.1.1 and Remark 12.1.4 in [3].

When the locally convex algebras are not multiplicatively convex you have to use the properly uniformly bounded approximate unit property we have introduced generalizing the well known uniformly bounded approximate unit property of locally multiplicatively convex algebras. This property is crucial to prove the Karoubi conjecture for arbitrary (not necessarily m-convex) Fréchet algebras. 


\section{References}

1. Arens, R.: The space $L^{\omega}$ and convex topological rings. Bull. Am. Math. Soc. 52, 931-935 (1946)

2. Bass, H.: Some problems in classical algebraic K-theory. In: Algebraic K-Theory II, Lecture Notes in Mathematics, vol. 342, pp. 1-73. Springer, New York, Berlin (1973)

3. Cortiñas, G.: Algebraic v. topological K-theory: a friendly match. In: Topics in Algebraic and Topological K-theory. Springer Lecture Notes in Mathematics (2008)

4. Cortiñas, G., Thom, A.: Comparison between algebraic and topological K-theory of locally convex algebras. Adv. Math. 218, 266-307 (2008)

5. Cortiñas, G., Haesemeyer, C., Weibel, C.: K-regularity, cdh-fibrant Hochschild homology and a conjecture of Vorst. J. Am. Math. Soc. 21, 547-561 (2008)

6. Gersten, S.: Higher K-theory of rings. Algebraic K-Theory I, Lecture Notes in Mathematics, vol. 341, pp. 1-40. Springer, New York, Berlin (1973)

7. Gubeladze, J.: Classical Algebraic K-Theory of Monoid Algebras, Lecture Notes in Mathematics, vol. 1437, pp. 36-94. Springer, New York, Berlin (1990)

8. Gubeladze, J.: K-theory of affine toric varieties. Homol. Homot. Appl. 1(5), 135-145 (1999)

9. Higson, N.: Algebraic K-theory of stable C*-algebras. Adv. Math. 67, 1-140 (1988)

10. Inassaridze, H.: Algebraic K-theory of normed algebras. K-Theory 21(1), 25-56 (2000)

11. Inassaridze, H.: Smooth K-groups for monoid algebras and K-regularity. Mathematics 3, 891-896 (2015)

12. Inassaridze, H.: Algebraic K-Theory. Kluwer Academic Publishers, The Netherlands (1995)

13. Inassaridze, H., Kandelaki, T.: K-theory of stable generalized operator algebras. K-Theory 27, 103-110 (2002)

14. Inassaridze, H., Kandelaki, T.: Smooth K-theory of locally convex algebras. Commun. Contemp. Math. 13(4), 553-577 (2011)

15. Inassaridze, H., Kandelaki, T.: La conjecture de Karoubi pour la K-théorie lisse. C. R. Acad. Paris, Sec. I 340 (2007)

16. Karoubi, M.: La périodicité de Bott en K-théorie générale. Ann. Sci. Ec. Norm. Sup. 4, 63-95 (1971)

17. Karoubi, M., Villamayor, O.: K-theorie algebrique et K-theorie topologique. J. Math. Scand. 28(2), 265-307 (1971)

18. Peimbert, H.A.: Matrix algebras and m-convexity. Demonstratio Mathematica 17(3), 711-722 (1984)

19. Quillen, D.: Higher algebraic K-theory I. In: Algebraic K-Theory I, Lecture Notes in Mathematics, vol. 341, pp. 77-139. Springer, New York, Berlin (1973)

20. Rolewicz, S., Zelazko, W.: Some problems concerning $B_{O}$ algebras. Tensor (New Series) 13, 269-276 (1963)

21. Rosenberg, J.: The algebraic K-theory of operator algebras. K-Theory 12(1), 75-99 (1997)

22. Rosenberg, J.: Comparison between algebraic and topological K-theory for Banach algebras and $\mathrm{C}^{*}$ algebras. In: Friedlander, E.M., Grayson, D. (eds.) Handbook of K-Theory, pp. 843-874. Springer (2004)

23. Schweitzer, L.B.: Dense m-convex Fréchet subalgebras of operator algebra crossed products by Lie groups. Int. J. Math. 4(4), 601-673 (1993)

24. Suslin, A., Wodzicki, M.: Excision in algebraic K-theory. Ann. Math. 136(1), 51-122 (1992)

25. Swan, R.G.: Some relations between higher K-functors. J. Algebra 21(1), 113-136 (1972)

26. Tillmann, U.: K-theory of fine topological algebras, Chern character and assembly. K-Theory 6(1), 57-86 (1992)

27. Trèves, F.: Topological Vector Spaces, Distributions and Kernels. Academic Press, New York, London (1967)

28. Wodzicki, M.: Excision in cyclic homology and in rational algebraic K-theory. Ann. Math. 120, 591639 (1989)

29. Wodzicki, M.: Algebraic K-theory and functional analysis. In: First European Congress of Mathematics (Paris 1992), Progress 120, vol. II, p. 485-496. Birkhauser, Basel (1994)

30. Zelazko, W.: On the locally bounded and m-convex topological algebras. Studia Mathematica 19, 333-356 (1960) 